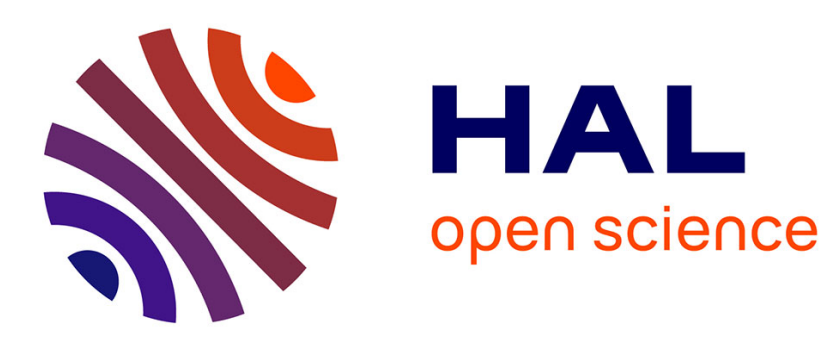

\title{
16O-12C scattering: description of the gross structure features using an optical model
}

\author{
A.J. Cole, N. Longequeue, J.F. Cavaignac
}

\section{To cite this version:}

A.J. Cole, N. Longequeue, J.F. Cavaignac. 160-12C scattering: description of the gross structure features using an optical model. Journal de Physique, 1977, 38 (9), pp.1043-1049. 10.1051/jphys:019770038090104300 . jpa-00208669

\section{HAL Id: jpa-00208669 https://hal.science/jpa-00208669}

Submitted on 1 Jan 1977

HAL is a multi-disciplinary open access archive for the deposit and dissemination of scientific research documents, whether they are published or not. The documents may come from teaching and research institutions in France or abroad, or from public or private research centers.
L'archive ouverte pluridisciplinaire HAL, est destinée au dépôt et à la diffusion de documents scientifiques de niveau recherche, publiés ou non, émanant des établissements d'enseignement et de recherche français ou étrangers, des laboratoires publics ou privés. 


\title{
Classification \\ Physics Abstracts 24.50 \\ ${ }^{16} \mathrm{O}-{ }^{12} \mathrm{C}$ SCATTERING : DESCRIPTION OF THE GROSS STRUCTURE FEATURES USING AN OPTICAL MODEL
}

\author{
A. J. COLE, N. LONGEQUEUE and J. F. CAVAIGNAC \\ Institut des Sciences Nucléaires, B.P. 257, 38044 Grenoble Cedex, France
}

(Reçu le 17 mars 1977, accepté le 16 mai 1977)

\begin{abstract}
Résumé. - La mesure de la diffusion de ${ }^{16} \mathrm{O}$ sur ${ }^{12} \mathrm{C}$ a été faite dans une région angulaire limitée dans les directions avant et arrière à des énergies laboratoires de ${ }^{16} \mathrm{O}$ de 80, 85, 91, 93, 95, 101 et $122 \mathrm{MeV}$. La fonction d'excitation du maximum de la distribution angulaire situé près de $156^{\circ}(\mathrm{c} . \mathrm{m}$.) a aussi été mesurée par pas de $\sim 0,5 \mathrm{MeV}$ entre 80 et $101 \mathrm{MeV}$ (lab.). Les résultats ont été ajoutés à d'autres résultats existants et la gross structure de l'évolution de la diffusion avec l'énergie a été analysée en utilisant un modèle optique dont le rayon imaginaire croît avec l'énergie.

Abstract. - Measurements of the scattering of ${ }^{16} \mathrm{O}$ on ${ }^{12} \mathrm{C}$ have been made over a limited angular range in the forward and backward hemispheres at laboratory $\left({ }^{16} \mathrm{O}\right)$ energies of $80,85,91,93,95,101$, $122 \mathrm{MeV}$. The excitation function of the maximum near $156^{\circ}$ (c.m.) has also been measured in steps of $\sim 0.5 \mathrm{MeV}$ between 80 and $101 \mathrm{MeV}$ (lab.). The data were combined with other existing measurements and the gross structure features analyzed using an optical model whose imaginary radius increases with energy.
\end{abstract}

$$
\left[\begin{array}{l}
\text { Nuclear reactions } \\
{ }^{12} \mathrm{C}\left({ }^{16} \mathrm{O},{ }^{16} \mathrm{O}\right){ }^{12} \mathrm{C}, E_{\text {lab }}=80-122 \mathrm{MeV} \\
\text { measured } \frac{\mathrm{d} \sigma}{\mathrm{d} \Omega}(\theta), \sigma(E),\left(\theta_{\text {c.m. }}=156^{\circ}\right) .
\end{array}\right]
$$

1. Introduction. - The ${ }^{12} \mathrm{C}-{ }^{16} \mathrm{O}$ system has, in recent years, been the subject of intense experimental and theoretical investigations mainly due to the existence of anomalously high backward angle crosssections exhibiting strong oscillations approximately described by $\left[P_{L_{s}}(\cos \theta)\right]^{2}$ where $L_{\mathrm{s}}$ is the angular momentum corresponding to an impact parameter characteristic of the surface of the interaction $[1,2,3]$. In addition for energies $E_{\mathrm{B}}<E_{\text {c.m. }}<30 \mathrm{MeV}\left(E_{\mathrm{B}}\right.$ is the Coulomb barrier $\approx 12 \mathrm{MeV}$ ) the backward angle cross-section fluctuates strongly with energy giving rise to the supposition of the existence of molecular resonances, especially at $E_{\text {c.m. }}=19.7 \mathrm{MeV}$ [2] and $E_{\text {c.m. }}=22.5 \mathrm{MeV}$ [1] where the influence of resonance like effects in reaction channels have been established [3,1]. At low energy $E_{\mathrm{B}}<E_{\text {c.m. }}<17 \mathrm{MeV}$ measurements of the total reaction cross-section [4] were explained using an optical model which however, possessed the unphysical characteristic of relatively small $(\sim 0.7)$ transmission coefficients for low partial waves.

One may question the wisdom of trying to fit fluctuations which, in this system, have a width of $\sim 0.5 \mathrm{MeV}$ with a gross structure model (optical model). Nevertheless by using $l$-dependent optical models some success has been obtained in fitting angular distributions at backward angles [5], $\left(^{1}\right)$. The physical origin of the $l$-dependence is however not clear and a number of explanations related either to the non formation of the compound nucleus [6] or to the angular momentum mismatch in direct reaction channels [7] have been proposed. Yet another possible origin was proposed by the authors of reference [5] who supposed that the $l$-dependent imaginary potential used in their analyses of ${ }^{16} \mathrm{O}-{ }^{12} \mathrm{C}$ scattering at 65 and $80 \mathrm{MeV}\left({ }^{16} \mathrm{O}\right.$ lab.) simulates coherent interference between the elastic scattering and the $\alpha$ transfer reaction. However, it remains to be established that such interference can reproduce the rapid fluctuations observed in excitation function measurements while preserving the regular oscillatory structure

(1) Charles, P., Private communication. 
observed at backward angles. Certainly, a single direct mechanism may be ruled out since such amplitudes vary too slowly with energy. This fact was emphasized by Devries [8] who limited his analysis of ${ }^{16} \mathrm{O}-{ }^{12} \mathrm{C}$ scattering in terms of the $\alpha$ transfer mechanism to the data at $80 \mathrm{MeV}$ where rapid fluctuations with energy were supposed to be less important.

Above $80 \mathrm{MeV}$ published data including angular distribution measurements is limited to forward angle distributions measured at $111 \mathrm{MeV}$ [9] and $168 \mathrm{MeV}$ [10]. It was therefore considered useful to extend the range of the data by measuring small portions of the forward and backward angle distributions between 80 and $120 \mathrm{MeV}$ (lab. ${ }^{16} \mathrm{O}$ ). The energy resolution available $\left(\Delta E / E \approx 10^{-3}\right)$ precluded a search for details of fluctuations in the excitation function although large effects, such as those observed at lower energies [2], if present, could be observed. Further, a verification of the presence of a backward angle rise in the angular distributions and a test of the simple $\left[P_{L_{s}}(\cos \theta)\right]^{2}$ behavior observed at lower energies was considered useful. Using the ${ }^{16} \mathrm{O}\left(5^{+}\right)$beam of the Grenoble ISN cyclotron we therefore measured small portions of the forward and backward angle distributions for ${ }^{16} \mathrm{O}-{ }^{12} \mathrm{C}$ scattering at laboratory energies of $80,85,91,93,95,101$ and $122 \mathrm{MeV}$. In addition, the excitation function of the maximum situated near $156^{\circ} \mathrm{c} . \mathrm{m}$. was measured between 80 and $101 \mathrm{MeV}$.

2. Experimental procedures. - The experiment was carried out at the ISN isochronous cyclotron of Grenoble. The experimental arrangement for the 80 to $101 \mathrm{MeV}$ data is shown in figure 1 . A collimated

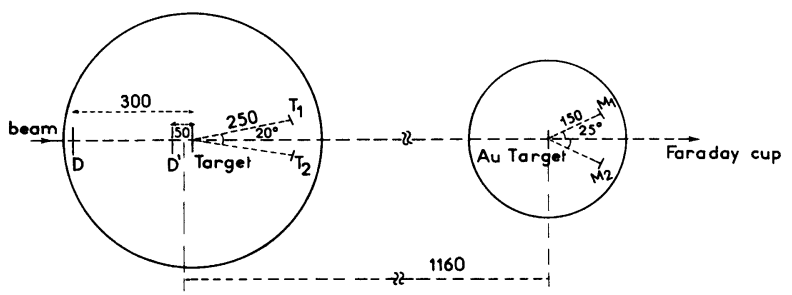

Fig. 1. - Experimental arrangement. Dimensions are given in millimetres.

beam of ${ }^{16} \mathrm{O}\left(5^{+}\right)$of up to $15 \mathrm{nA}$ (particle) was directed onto self supporting ${ }^{12} \mathrm{C}$ foils of thickness

$$
100 \pm 5 \mu \mathrm{g} / \mathrm{cm}^{2} \text {. }
$$

Two $\Delta E-E$ detector telescopes $\mathrm{T}_{1}$ and $\mathrm{T}_{2}$ mounted on a single movable arm, and separated by $20^{\circ}$ detected particles on the left and right sides of the beam. The angular aperture of the detectors were $0.5^{\circ}$ and $0.7^{\circ}$. The ${ }^{16} \mathrm{O}$ particles emitted in the forward direction and the recoiling ${ }^{12} \mathrm{C}$ particles (corresponding to backward angle ${ }^{16} \mathrm{O}$ in the c.m. system) were identified on line by a PDP9 data handling system [7].
The collimating system $\mathrm{DD}^{\prime}$ defined the beam direction to within $2^{\circ}$ which was not sufficiently precise for the performance of the experiment. Thus before each angular distribution run the angle between the beam direction and the chamber axis was measured using a $100 \mu \mathrm{g} / \mathrm{cm}^{2}$ gold target. This asymmetry angle was obtained when the ratio of the elastic counts in the left and right detectors was equal to the ratio of their solid angles. The constancy of the beam direction was then monitored continuously using a large gold target evaporated in a ${ }^{12} \mathrm{C}$ backing in a secondary chamber, the ratio between the count rates in two detectors $\left(\mathbf{M}_{1}\right.$ and $\left.\mathbf{M}_{2}\right)$ at $\pm 25^{\circ}$ with respect to this chamber axis indicating if any change took place in the beam direction. Data were accepted provided that the asymmetry angle remained constant to within $0.2^{\circ}$. As a further check the angular distributions were measured on both sides of the beam and the angular shift necessary for their superposition was compared to the initial asymmetry measured using the gold target. The agreement was always better than $0.2^{\circ}$ which was therefore assigned as the angular error to each data point. The quality of the identification spectrum did not permit us to rule out the presence of small contributions of other isotopes of $\mathrm{C}$ and $\mathrm{O}$. However because of negative reaction $Q$ values, contamination of the elastic peaks was due only to the presence of the ${ }^{13} \mathrm{C}$ target impurity $(1 \%)$ and was therefore neglected. A typical energy spectrum is shown in figure 2. The measured solid angle ratio between the two detection systems was checked by moving the arm completely to the left or to the right side of the beam and cross calibrating using the gold target.

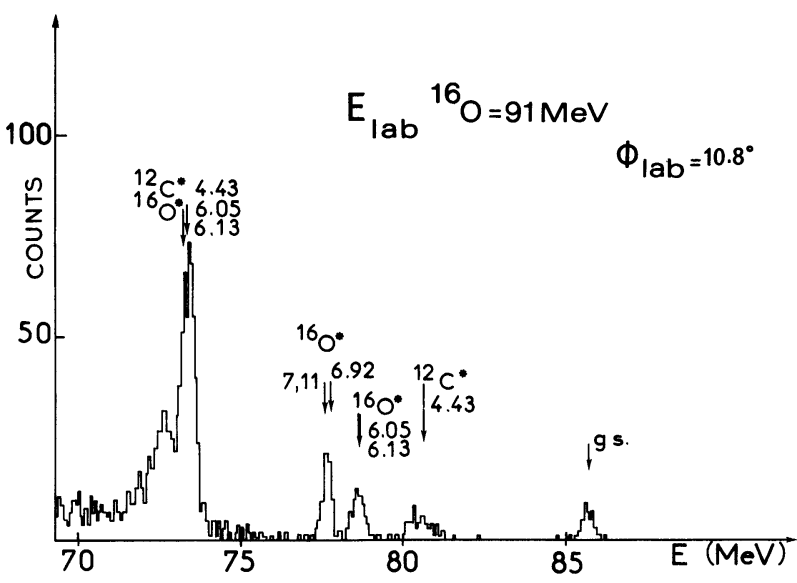

FIG. 2. $-{ }^{12} \mathrm{C}$ energy spectrum $\theta_{\text {lab }}=10.8^{\circ}$.

The beam energy was measured in a separate magnetic analyzing system to within $0.5 \mathrm{MeV}$. For energy changes of up to $5 \mathrm{MeV}$ aluminium foils mounted on a movable arm were used as degraders. The experimental energy resolution varied between $500 \mathrm{keV}$ and $1 \mathrm{MeV}$ depending on the degrader. With no degrader the $500 \mathrm{keV}$ resolution was principally due to kinematic 
effects. The forward angle elastic scattering measurement at $122 \mathrm{MeV}$ was performed in a larger chamber installed on the same beam line. For this experiment, the two detectors were mounted on separately movable arms. Otherwise the experimental arrangements were identical.

At forward angles the high count rate from the ${ }^{16} \mathrm{O}$ elastic could cause pile up problems in the ${ }^{12} \mathrm{C}$ channel. Anti-pile up devices were therefore included in the electronic set up and scalers were used to measure the raw $\Delta E$ count rates and the pile up rates. In addition a generator simulated ${ }^{12} \mathrm{C}$ peak was fed through each system and was used to make dead time corrections. Any gain changes in the electronics could thus also be detected.

3. Results and data analysis. - In contrast to the results at lower energies our data exhibit a nearly monotonic decrease in the magnitude of the backward angle cross-section as the energy increases. Fluctuations of width less than $500 \mathrm{keV}$ if present, were obscured by the experimental energy resolution. Further and rather surprisingly, although the back angle differential cross-sections exhibit deep oscillations the maxima and minima do not move progressively towards $180^{\circ} \mathrm{c} . \mathrm{m}$. as would be expected from a $\left[P_{L_{s}}(\cos \theta)\right]^{2}$ description. The forward angle data present no such anomaly. Since no rapid variations with energy were observed, it was thought reasonable to try and fit the positions of the back angle maxima with a potential model. Initially, a shallow $l$-dependent potential used by Badawy et al. [11] in an attempt to fit the $166^{\circ}$ excitation function between 15 and $30 \mathrm{MeV}$ (c.m.), was tried but failed to fit the anomalous back angle behaviour. By adjusting the l-dependence parameters at each energy good fits could be obtained but no simple energy dependence of the model was apparent."

Recently, von Oertzen [12], has remarked on the success of deep potentials given for example by the double folding model [13] in accounting for the systematics of heavy ion transfer reactions. It was therefore decided to try a deep potential of the (conventional) form :

$$
\begin{array}{r}
V_{\text {opt }}=\frac{V_{\mathbf{R}}}{1+\exp \left(\frac{r-R_{0}}{a}\right)}+\frac{W_{\mathbf{V}}}{1+\exp \left(\frac{r-R_{\mathrm{I}}(E)}{a}\right)}+ \\
+V_{\mathrm{c}}\left(r, R_{\mathrm{c}}\right) .
\end{array}
$$

The Coulomb potential $V_{\mathrm{c}}\left(r, R_{\mathrm{c}}\right)$ was that due to a charged sphere of radius $2.86 \mathrm{fm}$. The value of the radius parameter has been justified in reference [14]. The depth of the real part was chosen following Satchler [15] as roughly half that given by the double folding model. The geometry parameters were determined by fitting the variation with energy of the positions of the backward angle maxima. An excellent fit was obtained with the values given in table I
TABLE I

\begin{tabular}{|c|c|c|c|c|}
\hline $\begin{array}{c}V_{\mathrm{R}} \\
\mathrm{MeV}\end{array}$ & $\begin{array}{l}r_{0}^{*} \\
\mathrm{fm}\end{array}$ & $a$ & $\begin{array}{c}W_{\mathbf{V}} \\
\mathrm{MeV}\end{array}$ & $\begin{array}{l}r_{\mathrm{I}}^{*} \\
\mathrm{fm}\end{array}$ \\
\hline - & - & - & - & - \\
\hline 430 & 1.40 & 0.79 & 28.0 & $\begin{array}{l}0.0175 E \text { (lab.) } \\
-10^{-9} E^{4} \text { (lab.) }\end{array}$ \\
\hline
\end{tabular}

Optical model parameters

${ }^{*}$ ) The potential radii are obtained by multiplying by $A_{1}^{1 / 3}$ where $A_{1}=12$.

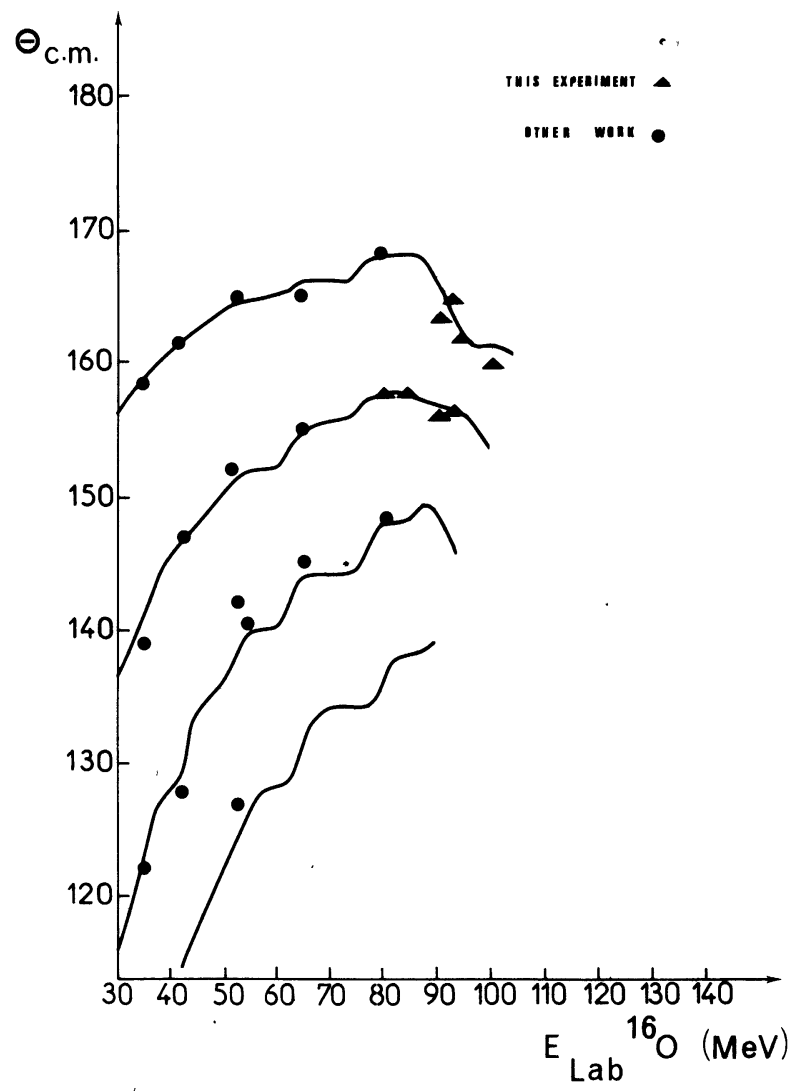

FIG. $3-{ }^{16} \mathrm{O}-{ }^{12} \mathrm{C}$ scattering. Variations of the positions of the back angle maxima with energy. The solid lines extending up to $\sim 100 \mathrm{MeV}$ correspond to calculations made with the potential parameters of table I. Above this energy the simple oscillatory structure of the backward angle distributions is lost and the evolution of a particular maximum is thus impossible to follow.

and is displayed in figure 3 . Remarkably these parameters imply a mean square radius of the real potential $\left\langle r^{2}\right\rangle_{\mathrm{v}}=14.8 \mathrm{fm}^{2}$ which is almost exactly that given by the double folding model prescription

$$
\begin{aligned}
\left.\left\langle r^{2}\right\rangle_{\mathrm{V}} \text { (folding model }\right) & = \\
= & \left\langle r^{2}\right\rangle_{\rho 1}+\left\langle r^{2}\right\rangle_{\rho 2}+\left\langle r^{2}\right\rangle_{\mathrm{d}}
\end{aligned}
$$

where $\left\langle r^{2}\right\rangle_{\rho 1}$ and $\left\langle r^{2}\right\rangle_{\rho 2}$ are the mean square radii of the densities of the interacting particles and $\left\langle r^{2}\right\rangle_{\mathrm{d}}$, that of a phenomenological nucleon-nucleon force. Taking the mean square radii of the densities from electron scattering measurements [16] and that of the two body force from reference [17] gives a value of $\left\langle r^{2}\right\rangle_{\mathrm{V}}$ (folding model $)=14.84 \mathrm{fm}^{2}$. 
The predictions of figure 3 were shown to be sensitive to the values of $a$ and $R_{0}$ independently. This sensitivity was not preserved when fits to the positions of the forward angle minima were made. These positions which, as can be seen in figure 4, were well fitted by the parameter set given in table I were shown to be determined uniquely by the value of $\left\langle r^{2}\right\rangle_{\mathrm{v}}$. The broken lines in figure 4 give some idea of the sensitivity to this parameter. It was verified that variations in the imaginary potential parameters have little effect on these conclusions. At any one energy variations in either the depth or the geometry parameters were shown to affect chiefly the depths of forward angle minima and the magnitude of the backward angle differential cross-section.

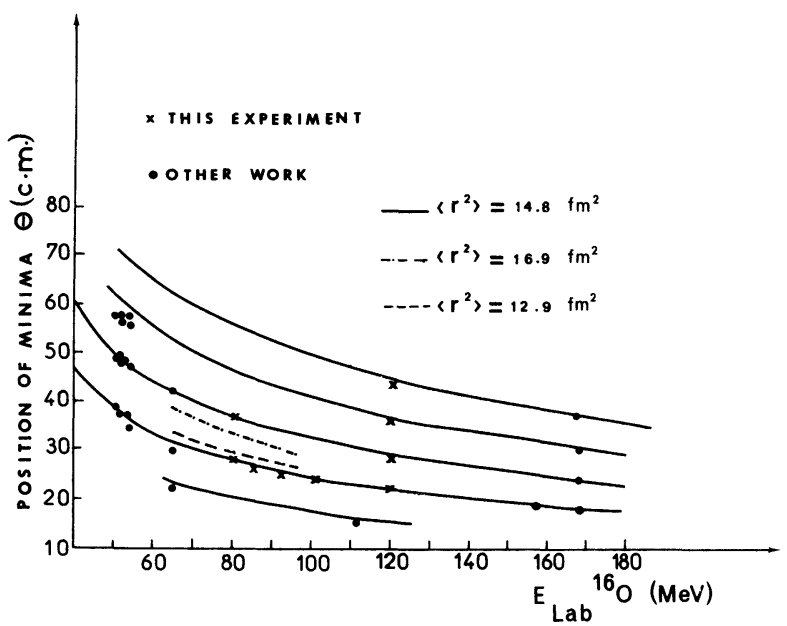

FIG. 4. $-{ }^{16} \mathrm{O}-{ }^{12} \mathrm{C}$ scattering. Variations of the positions of forward angle minima with energy. The solid lines correspond to calculations made with the potential parameters of table I.

The determination of the imaginary potential (table I) was carried out as follows. Below $33 \mathrm{MeV} \mathrm{c.m.}$ a linear increase of the radius parameter was found by comparing the excitation function data of Badawy et al. [11] with the optical model predictions. However, since this data exhibits rapid fluctuations with energy it was deemed necessary to define an experimental energy averaged cross-section at each energy $E_{0}$ through the relation

$$
\begin{aligned}
\frac{\partial \sigma}{\partial \Omega}\left(E_{0}\right)=\frac{1}{\Delta E \sqrt{2 \pi}} \int_{15}^{34} \frac{\mathrm{d} \sigma}{\partial \Omega}\left(E_{\text {c.m. }}\right) \times & \\
& \times \exp \frac{\left(E_{\text {c.m. }}-E_{0}\right)^{2}}{2 \Delta E^{2}} \mathrm{~d} E_{\text {c.m. }} .
\end{aligned}
$$

with $\Delta E=1.0 \mathrm{MeV}$. The linear part of the function $r_{\mathrm{I}}(E)$ given in table I was thus obtained by demanding a good overall fit to the magnitude of this energy averaged cross-section. As can be seen in figure 5 the energy averaged data reveal gross structure resonances which are reproduced by the calculation. This structure was shown to be independent of $\Delta E$ for

$$
0.5 \mathrm{MeV}<\Delta E<2.5 \mathrm{MeV} \text {. }
$$

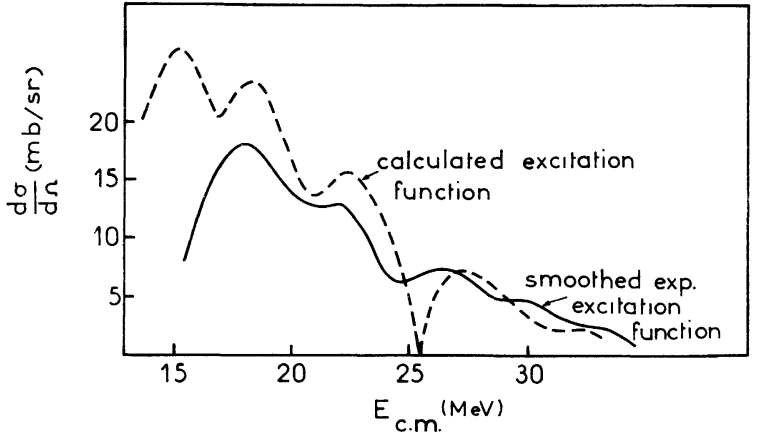

FIG. 5. $-{ }^{16} \mathrm{O}-{ }^{12} \mathrm{C}$ scattering. Energy averaged excitation function (solid line) for the maximum situated near $160^{\circ}$ (c.m.) calculated from the data of Badawy et al. [11] as described in the text. The optical model prediction (dotted line) was made using the parameters given in table I. Since the experimental data exist only on the range 15 to $34 \mathrm{MeV}$ c.m., the averaged cross-section is incorrect in $\sim 2 \mathrm{MeV}$ wide regions at the beginning and end of this range.

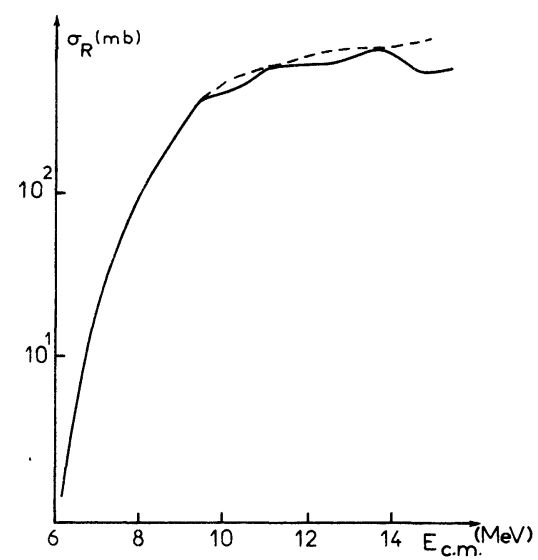

FIG. 6. $-{ }^{16} \mathrm{O}-{ }^{12} \mathrm{C}$ scattering. Variation of total reaction crosssection with energy [4] (full line) compared with the optical model prediction made using the parameters of table I.

As a further check we compared the prediction made using these parameters with the total reaction crosssection data of Kuenher and Almquist [4]. Figure 6 shows that excellent agreement was obtained.

Above $30 \mathrm{MeV}$ c.m. it was found necessary to add a non linear term to the function $r_{\mathrm{I}}(E)$ in order to reproduce the absolute value of the back angle differential cross-section at $52.3 \mathrm{MeV}$ c.m. (Fig. 11). When this was done a good fit to our excitation function measured between 34 and $41 \mathrm{MeV}$ c.m. was obtained (Fig. 7). Further, the maximum value of $r_{\mathrm{I}}(E)(2.15 \mathrm{fm}$ at $70 \mathrm{MeV}$ c.m.) gives an excellent fit to the forward angle scattering data of Hiebert and Garvey [10] (Fig. 8). At low energies the influence of the non linear term is negligible.

Since a smooth monotonic increase of the imaginary potential radius parameter gives a good description of all data considered it would be tempting to try to interpret this comportment in terms of some physical model. However a number of problems preclude such speculation. The most important is the existence of ambiguities in the imaginary potential. Thus, in the tail of the potential a linear increase in the radius 


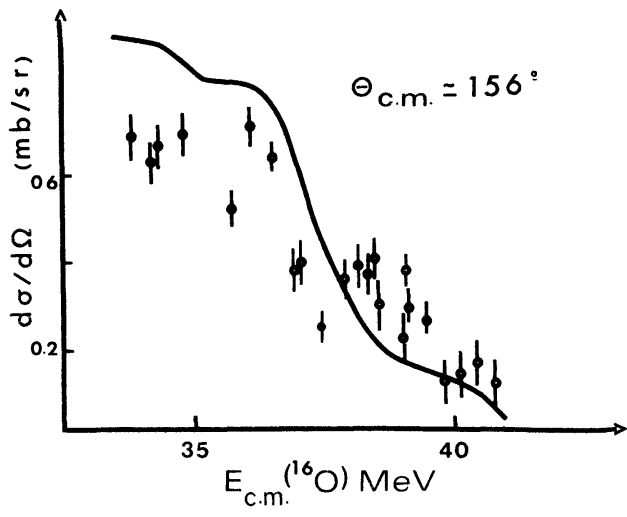

FIG. 7. $-{ }^{16} \mathrm{O}-{ }^{12} \mathrm{C}$ scattering. Prediction of excitation function for the maximum situated near $156^{\circ} \mathrm{c} . \mathrm{m}$. The data points are those measured in the present work.

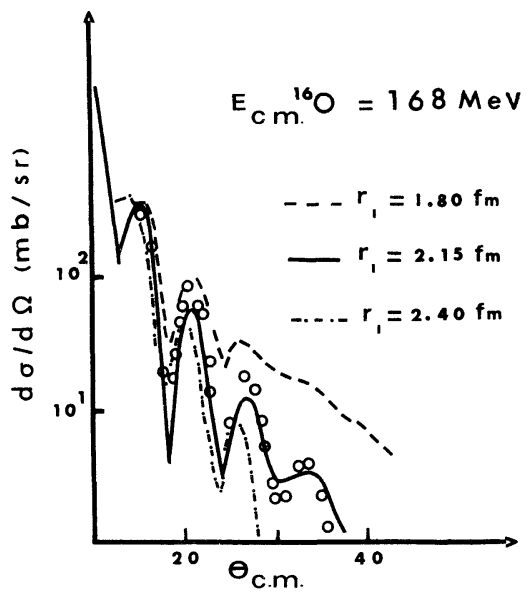

Fig. 8. $-{ }^{16} \mathrm{O}-{ }^{12} \mathrm{C}$ scattering. $168 \mathrm{MeV}$ (lab.) data of ref. [10]. The prediction (full line) was made with the maximum value of $r_{1}(E)$ as explained in the text. Other predictions (dotted lines) are shown to give an idea of the sensitivity to $r_{\mathrm{I}}(E)$. Other parameters are as given in table $\mathrm{I}$.

parameter with energy may be replaced by an exponential increase in the potential depth. Also a first order Taylor expansion indicates that the addition of a derivative Saxon Woods term, whose depth increases linearly with energy, to an energy independent volume Saxon Woods form is equivalent, over short energy ranges, to a single volume term with a linearly increasing radius parameter. Calculations made with these forms over small energy ranges verified these equivalences and thus underlined the phenomenological ambiguity. Even if no such ambiguity existed a difficulty would arise since one expects the optical model to underestimate the average of a rapidly fluctuating cross-section by the average of the cross-section due to the fluctuating part of the scattering wave function. Since the origin of the observed fluctuations is, at present, unknown it is impossible to decide how much of the energy averaged cross-section should be allocated to potential scattering.
Finally we remark on the fact that our parameters suffer from the same drawback as those of Kuenher and Almquist [4] in as much as at low energies they imply non zero values of $\left|\eta_{l}\right|$ for low partial waves. In order to investigate this point a strongly absorbing core was added to our optical potential the parameters of which are given in figure 9. The small diffuseness was chosen to minimise the effect of the core in the surface region while avoiding the introduction of sharp discontinuities in the potential. As can be seen in figure 9 (inset), increasing the depth of this term

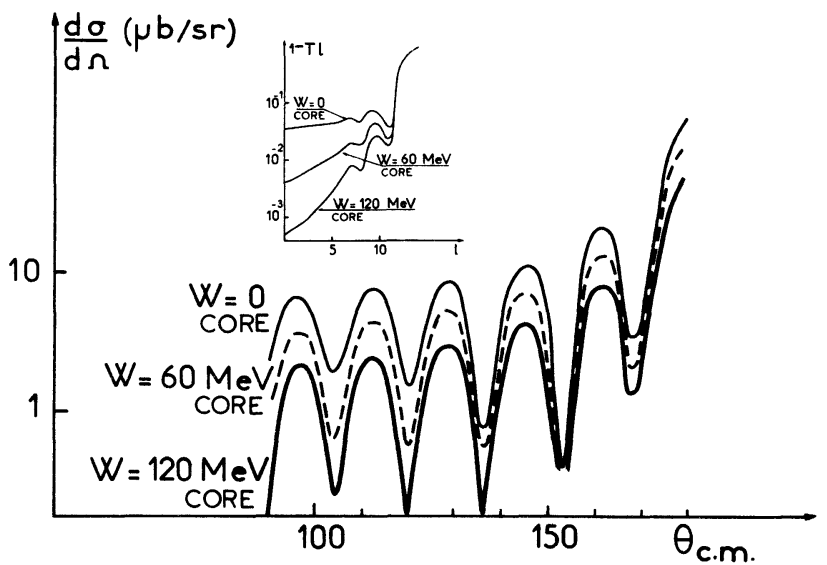

Fig. 9. $-{ }^{16} \mathrm{O}-{ }^{12} \mathrm{C}$ scattering. Sensitivity of the calculated $17.2 \mathrm{MeV}$ (c.m.) angular distribution to the addition of a strongly absorbing core to the imaginary potential of table I

$$
W_{\mathrm{V} \mathrm{CORE}}(r)=\frac{W_{\mathrm{CORE}}}{1+\exp \frac{r-R_{\mathrm{CORE}}}{a_{\mathrm{CORE}}}}
$$

with $R_{\mathrm{CORE}}=0.5 A_{1}^{1 / 3} \mathrm{fm} .\left(A_{1}=12\right), a_{\mathrm{CORE}}=0.3 \mathrm{fm}$. The effect of the core on the transmission coefficients $T_{l}$ is shown as an inset.

has a large effect on the transmission coefficients for low partial waves while producing no significant change in the partial waves scattered from the potential surface. Further, it is clear from the figure, that the large changes in the transmission coefficients for low partial waves do not strongly affect the back angle differential cross-sections. We thus conclude that the non physical character of the $\left|\eta_{l}\right|$ values for low partial waves is associated with the use of the Saxon Woods imaginary potential form factor and that this form is probably not appropriate for use in analysis of light heavy ion scattering.

Predictions for the back angle differential crosssections are shown with the data in figure 10 and, for the more complete data at $122 \mathrm{MeV}$ in figure 11 . No detailed fitting was attempted because of the limited angular range of the data.

4. Discussion. - Contrary to the statement made in the introduction of reference [5] it is possible to fit both forward and backward angle ${ }^{16} \mathrm{O}-{ }^{12} \mathrm{C}$ scattering simultaneously using a simple potential model. The main question remains that of determining whether 


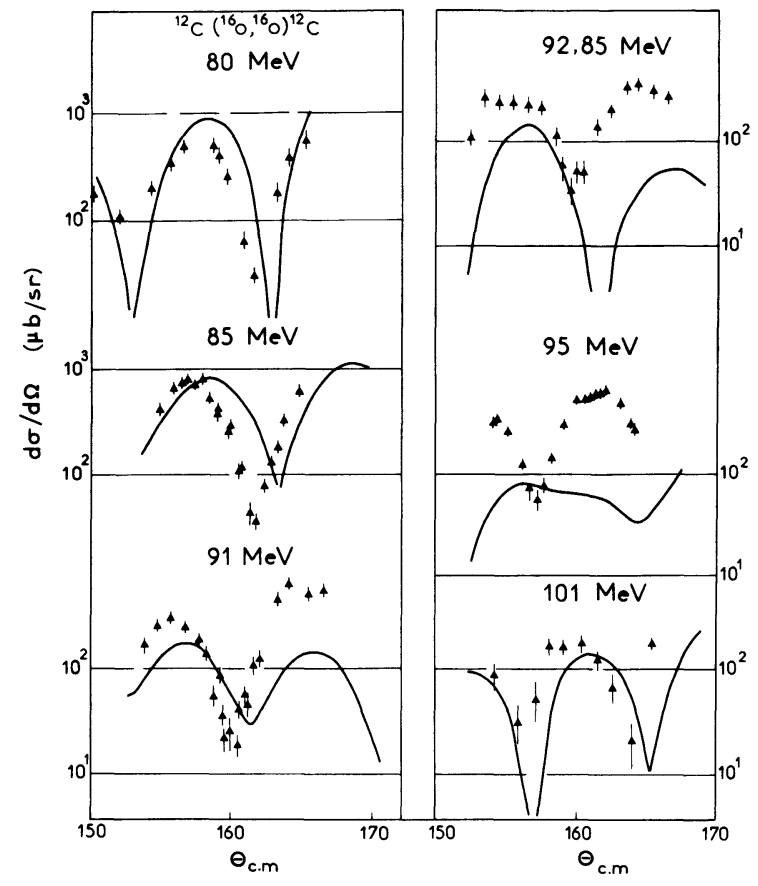

FIG. $10 .-{ }^{16} \mathrm{O}-{ }^{12} \mathrm{C}$ scattering. Back angle data between 80 and $100 \mathrm{MeV}$ (lab.) together with optical model predictions made using the parameters given in table I.

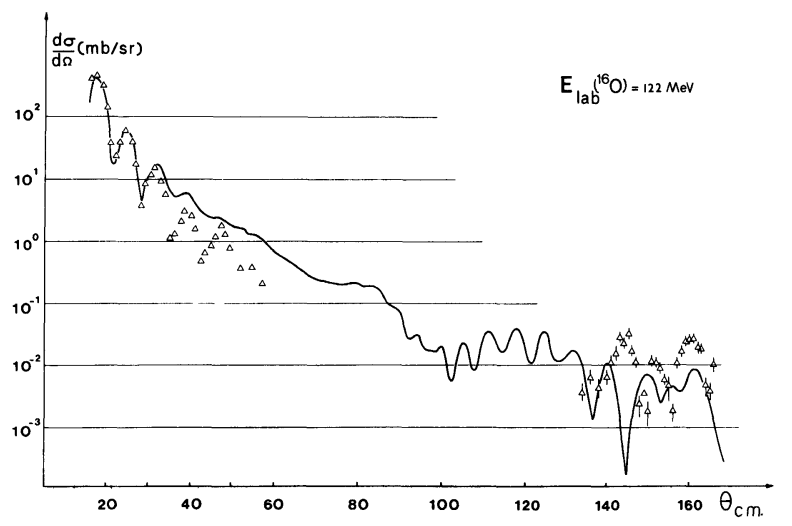

FIG. 11. $-{ }^{16} \mathrm{O}-{ }^{12} \mathrm{C}$ scattering. Data at $122 \mathrm{MeV}$ with the optical model prediction made using the parameters given in table I.

or not the deep potential found in the present analysis has physical significance. The lack of sensitivity of the predictions to the presence of a strongly absorbing core (Fig. 9) indicates that the interior part of the potential at radii $<1.5 \mathrm{fm}$ is not well determined by the data considered. However at distances greater than this value a deep potential would seem to be indicated. Further, our analysis shows that the geometry parameters of such a potential are well determined provided that data at forward and backward angles over a large energy range are considered.

Theoretical calculations [18, 19] of the ion-ion potential usually predict a shallow potential such as that used in the analysis of the ${ }^{12} \mathrm{C}-{ }^{12} \mathrm{C}$ data by the Yale group [20]. However, a recent calculation of the ${ }^{16} \mathrm{O}-{ }^{16} \mathrm{O}$ potential [21] using a generator coordinate method produced a potential rather like that used in the present analysis. Detailed comparison is difficult since the calculation of the interior and exterior parts of the potential were made in different limits of the approximations employed. We note however that the authors predict a potential of $\sim 430 \mathrm{MeV}$ central depth falling to half of this value for radii between 2 and $4 \mathrm{fm}$. They also conclude that the surface of the potential is well approximated by the double folding model.

From a phenomenological point of view our results agree with the findings of Kozab et al. [22] who, in analyzing the variation of complete fusion crosssections with energy for ${ }^{27} \mathrm{Al}+{ }^{16} \mathrm{O},{ }^{27} \mathrm{Al}+{ }^{20} \mathrm{Ne}$ and ${ }^{27} \mathrm{Al}+{ }^{32} \mathrm{~S}$, were led to the conclusion that the real part of the optical potential was strongly attractive at radii considerably smaller than the sum of the radii of the interacting particles. Analysis of systematics of transfer reactions by von Oertzen [13] also indicated the necessity of a deep real potential. On the other hand there exist many phenomenological analyses of ion-ion scattering using shallow real potentials [24]. It is our opinion that such analyses are strongly influenced by the choice of radius parameter which is almost always taken to be the sum of the radii of the interacting nuclei. A priori of course there is no reason for this choice which, at least for light targets, disagrees with the supposition that the potential surface is well represented by the double folding model. For example in the ${ }^{16} \mathrm{O}^{12} \mathrm{C}$ case the sum of the radii is

$$
R \sim 1.25\left(12^{1 / 3}+16^{1 / 3}\right)=6.0 \mathrm{fm} .
$$

Even for zero diffuseness we obtain

$$
\left\langle r^{2}\right\rangle_{\mathrm{v}}=\frac{3}{5} R^{2}=21.5 \mathrm{fm}^{2}
$$

which may be compared with the value of $14.8 \mathrm{fm}^{2}$ given by the double folding model (section 3 ).

A somewhat surprising result of our analysis is the smallness of the imaginary potential. Its main justification comes from the agreement between the calculated and experimental reaction cross-sections as well as the overall agreement with the magnitudes of the backward angle differential cross-sections. Because of the ambiguities detailed in the previous section we may conclude only that the imaginary potential increases with energy. This conclusion may of course be understood partly in terms of the increasing density of states in the ${ }^{28} \mathrm{Si}$ compound nucleus and partly in terms of the availability of an increasing number of direct and semi-direct reaction channels with increasing energy.

Finally, we should remark on possible contributions from the $\alpha$ transfer mechanism. Our analysis has shown that all the gross structure features of the data are well described by a single direct process (elastic scattering). The $\alpha$ transfer reaction, which may be an alternative method of describing the back angle 
part of the cross-section, would be expected to be characterized by a $\left[P_{L_{s}}(\cos \theta)\right]^{2}$ behaviour which disagrees with observation above $34 \mathrm{MeV}$ (c.m.). Below this energy it is not clear whether the interference of these mechanisms is capable of producing the observed fluctuations but this possibility should not be ruled out. Detailed calculations, clearly necessary to test this point, are in progress.
Acknowledgments. - The experiments described in this paper were carried out in collaboration with members of a group of physicists of C.E.N. Saclay. We wish to thank them for their assistance. Our thanks are especially due to Dr's P. Charles and B. Fernandez for many fruitful discussions and to Professor J. P. Longequeue (ISN) for experimental assistance and for careful reading of the manuscript.

\section{References}

[1] Charles, P., Badawy, I., Berthier, B., Dost, M., Fernandez, B., Gastebois, J. and Lee, S. M., Proceedings of the International Conference on Nuclear Physics, Vol. 1, edited by J. de Boer and H. J. Mang (North-Holland, Amsterdam) 1973, p. 539.

[2] Malmin, R. E., Siemssen, R. H., Sink, D. A. and Singh, P. P., Phys. Rev. Lett. 28 (1972) 1590.

[3] Stokstad, R., Shapira, D., Chua, L., Parker, D., Sachs, M. W., Wieland, R. and Bromley, D. A., Phys. Rev. Lett. 28 (1972) 1523.

[4] Kuenher, J. A. and Almquist, E., Phys. Rev. 134B (1964) 1229.

[5] Gutbrod, H. H., Bock, R., Von Oertzen, W. and Schlotthauer-Voos, U. C., Z. Phys. 262 (1973) 377.

[6] Cohen, S., Plasil, F. and Swiatecki, W. J., Ann. Phys. 82 (1974) 557

[7] Chatwin, R. A., Eck, J. S., Robson, D. and Ritcher, A., Phys. Rev. C 1 (1970) 795.

[8] Devries, R. M., Nucl. Phys. A 212 (1973) 207.

[9] Williams, D. J. and Siegert, F. E., Nucl. Phys. 30 (1962) 373.

[10] Hiebert, J. C. and Garvey, G. T., Phys. Rev. 135B (1974) 346.

[11] Badawy, I., Berthier, B., Charles, P., Fernandez, B. and GasteboIS, J., Note CEA-N-1791 (1974) p. 14.

[12] Von Oertzen, W., Preprint LBL 1985 (1973).
[13] EIsen, Y., Phys. Lett. 37B (1971) 33.

[14] IWE, H., WiEBICKe, H. J., European Conference on Nuclear Physics with Heavy Ions, Caen 1976, Communications p. 20.

[15] SATCHLeR, G. R., In International Conference on Reactions Between Complex Nuclei, edited by R. L. Robinson, F. K. McGowan, J. B. Ball and J. H. Hamilton (NorthHolland, Amsterdam) 1974, vol. 2, p. 171.

[16] Hofstader, R., Ann. Rev. Nucl. Sci. 7 (1957) 308.

[17] Greenlees, G. W., Pyle, G. J. and Tang, Y. C., Phys. Rev. $171(1968) 1115$.

[18] Brink, D. M. and Stancu, F. L., Nucl. Phys. A 243 (1975) 175.

[19] Brueckner, K. A., Buchler, J. R. and Kelly, M. M., Phys. Rev. 173 (1968) 944.

[20] Bromley, D. A., In Nuclear Reaction induced by Heavy Ions, Proc. International Conference, Ed. R. Bock, W. R. Hering (Heidelberg) 1969, p. 49.

[21] Jaqaman, H. R. and Mekjian, A. Z., Nucl. Phys. A 259 (1976) 157.

[22] Kozub, R. L., Lu, N. H., Miller, J. M., Logan, D., Debiak, T. W. and Kowalski, L., Phys. Rev. 11C (1975) 1497.

[23] See for example Heavy ion scattering. Proceedings of the Symposium at Argonne National Laboratory, ANL 7837. 